\title{
THE ROLE OF BETA-2 ADRENERGIC RECEPTOR GENE POLYMORPHISM IN THE PATHOPHYSIOLOGY OF ASTHMA ASSOCIATED WITH OBESITY
}

\author{
Ibrahim M El Akkary ${ }^{(1)}$, Mervat E El Seweify ${ }^{(2)}$, Mohamed Mokhtar Mohamed $^{(3)}$, \\ Mamdouh M El -Yamany ${ }^{(4)}$, Eman Y. Khairy ${ }^{(5)}$, Ola A. Salama ${ }^{(5)}$ \\ (1, 2, 4, 5, 6) Department Clinical Pulmonary Physiology unit, Human Physiology, ${ }^{(3)}$ Department Human \\ Genetics, Medical Research Institute, Alexandria University, Egypt
}

\begin{abstract}
:
Background and objectives:: Several mechanisms have been suggested to explain the association between asthma and obesity, one of them is the presence of common genetic predictors as $\beta 2$ adrenergic receptor gene polymorphism. To test this suggestion, the present study was carried out.Subjects: 60 asthmatic patients (50 females and10 males) and 60 normal subjects (46 females and 14 males) were included in this study. Methodology: measurement of body weight and height, waist circumference and waist to hip ratio. Asthma severity was assessed by disease severity score. Pulmonary function tests and bronchial reactivity by methacholine inhalation challenge $\left(\mathrm{PD}_{20-\mathrm{FEV} 1}\right)$ were performed and DNA extraction, Polymerase chain reaction (PCR)and restriction digestion was performed at codons 16 and 27 of $\beta 2 A R$ to determine $\beta 2$ adrenergic receptor gene polymorphism. Results: No significant difference was detected between the asthmatic and control subjects as regards allelic or genotype frequencies of $\beta 2 \mathrm{AR}$ gene at codon 16 and codon 27 and no significant difference was found as regards genotype frequencies of $\beta 2 \mathrm{AR}$ gene at codon 16 and codon 27 between obese and non-obese asthmatics. A significantly increased frequency of Gly/Gly at codon 16 was found among severe asthmatics in comparison with mild and moderate asthmatics. A significantly higher frequency of genotype Glu/Glu at codon 27 was detected among obese subjects when compared to non-obese subjects. There was a significant difference in FEV1\% predicted and in FEF $25-75 \%$ predicted in non- obese asthmatics when distributed according to genotypes at codon 16 . No significant difference in the distribution of the asthmatics according to the value of PD20FEV1 among genotypes of B2AR gene at codon 16, codon 27 was found.

Conclusion: Arg16Arg was found to be protective from development of severe asthmaGlu27Gluwassignificantly associated with obesity. However, there was no detectable specific genotype for the association of asthma and obesity.
\end{abstract}

Keywords: Asthma; $\beta 2$ adrenergic receptor gene polymorphism; Obesity.

\section{INTRODUCTION}

A sthma and obesity are prevalent disorders, each with a significant public health impact. ${ }^{(1)}$ Epidemiologic data indicate that obesity increases the prevalence and Incidence of asthma and reduces asthma control. ${ }^{(2)}$
Several mechanisms have been suggested to explain the association between asthma and obesity including mechanical effects of obesity on pulmonary physiology, systemic inflammation, co-morbidities of obesity, prenatal diet and nutrition, hormonal factors and common genetic predictors. ${ }^{(3-7)}$
Some studies ${ }^{(8,9)}$ that have focused on either asthma or obesity have identified genes, including angiotensin I-converting enzyme (ACE), adrenergic receptor $\beta-2$ (ADRB2), and vitamin $D$ (1,25 dihydroxyvitamin $\mathrm{D} 3$ ) receptor (VDR) that might influence both diseases. Genes such as leptin(LEP); protein kinase $\mathrm{C}$ alpha (PRKCA); and tumor necrosis factor (TNF) have also been evaluated for pleiotropic effects that influence both asthma and obesity simultaneously. ${ }^{(10-12)}$

Comparative linkage analyses of asthma and obesity show an overlap of chromosomal regions (chromosomes $5 q, 6 p, 11 q$ and $12 q$ ) linked to both conditions. $^{(13)}$ Chromosome $5 q$ contains the gene ADRB2, which codes for the $\beta$-2-adrenergic receptor that exerts effects on the sympathetic nervous system and in baseline metabolism; its pharmacological activation leads to bronchodilation of the airways. ${ }^{(14)}$

The ADRB2 is expressed on airway smooth muscle (ASM), inflammatory cells, and adipose tissue, where endogenous catecholamines influence energy expenditure, airway tone, and airway inflammation. ${ }^{(15)}$ Furthermore, insulin levels, which are altered in obesity, can influence $\beta 2$-adrenergic receptor sensitivity. ${ }^{(16)}$ The ADRB2 is involved in lipid mobilization, as a major lipolytic receptor in human fat cells, and genetic variation in this receptor gene could theoretically reduce lipolysis and predispose to obesity.

The most frequent single-nucleotide polymorphisms occur at codon 16 and codon 27. ${ }^{(18)}$ Accordingly, 
the aim of the present work is to study the possible role of beta 2 adrenergic receptor gene polymorphism in the pathophysiology of the association between obesity and asthma.

\section{SUBJECTS AND METHODS}

\section{Study Design}

The study was carried out in 3 sessions on three separate days:

During the first session full history taking was done with anthropometric measurements (BMI, waist circumference and waist/hip ratio)and exclusion criteria were applied according to ATS guidelines for Spirometry and Methacholine challenge testing. Training on Spirometry was carried out.

During the second session Spirometry, Methacholine, questionnaire to assess severity and sampling were carried out .

During the third session molecular studies were done.

\section{SUBJECTS}

The study included 120 adults. 60 asthmatic patients (50 females and10 males) as defined by the American Thoracic Society ${ }^{(19)}$ and 60 normal subjects (46 females and 14 males) as controls were included in this study in the period from January 2010 to April 2011. Both patients and control subjects were selected and grouped according to their Body Mass Index (BMI) into nonobese group, $\mathrm{BMI} \leq 25.00 \mathrm{~kg} / \mathrm{m} 2$ and obese group, $\mathrm{BMI} \geq 30.00$ $\mathrm{kg} / \mathrm{m} 2$.

The four groups (each group includes 30 subjects)are:

- Obese asthmatics

- Non-obese asthmatics

- Obese control subjects

- Non-obese control subjects

\section{METHODS}

1. Assessment for asthma severity. ${ }^{(20)}$

2. Asthma severity was assessed by Disease Severity Score (DSS). ${ }^{(20)}$

3. Physiological studies

The following parameters were assessed for both patients and normal subjects:

a) Pulmonary function tests including Forced vital capacity (FVC),Forced expiratory volume in one second (FEV1), FEV1/FVC\% and Forced expiratory flow rate between $25 \%$ and $75 \%\left(\mathrm{FEF}_{25 \%-75 \%}\right)$ were measured using computerized dry spirometer (Jaeger, Germany) with automatic dosimeter for methacholine inhalation challenge. All measurements were performed according to the European Respiratory Society and American Thoracic Society protocol for spirometry standardization. ${ }^{(21)}$ b) Determination of bronchial reactivity by methacholine inhalation challenge (only in asthmatic subjects) using the five-breath dosimeter protocol according to the guidelines of The American Thoracic Society for methacholine inhalation challenge test. ${ }^{(22)}$

\section{Molecular studies}

A) DNA extraction:

Two milliliter of whole venous blood were collected from each subject on vaccutainer EDTA tube under complete aseptic technique. Genomic DNA was extracted from whole blood by DNA salting out technique. ${ }^{(23)}$

\section{B) Polymerase Chain Reaction (PCR)}

The Arg16Gly and Gln27Glu in the $\beta 2 A R$ gene were amplified using the following gene-specific primers: ${ }^{(24-26)}$ The forward primer:5-GGC-CCA-TGA-CCA-GAT-CAG-CA-3 The reverse primer: 5-GAA-TGA-GGC-TTC-CAG-GCG-TC-3

The forward primer: CTT-CTT-GCT-GGC-ACG-CAA-T-3

The reverse primer:CCA-GTG-AAG-TGA-TGA-AGT-AGT-TGG-3

The forward primer: 5-GCC-TTC-TTG-CTG-GGC-ACC-CAT-3

The antisense primer:5-CAT-ACG-CTC-GAA-CTT-GGC-CAT-C-3

DNA amplification was performed in a Thermohybraid PCR Express Thermacycler, with a total reaction volume of 25 ul containing:

Milli Q water: $12.5 \mathrm{ul}$

DNA: $300 \mathrm{ng}$

Forward primer (Bioron): 20 Pico moles

Reverse primer (Bioron): 20 Pico moles

PCR master mix: 12.5 ul Amplification was performed using initial denaturation at $95^{\circ} \mathrm{C}$ for 3 minutes

followed by 35 cycles of $94^{\circ} \mathrm{C}$ for 30 seconds, $66^{\circ} \mathrm{C}$ for 30 seconds and $72^{\circ} \mathrm{C}$ for 30 seconds with a final extension of $70^{\circ} \mathrm{C}$ for 6 minutes.

Following amplification, 9 ul of the PCR product were mixed with 2 ul of $6 \times$ loading buffer $(0.09 \%$ bromophenol blue, $0.09 \%$ xylene cyanol, $60 \mathrm{Mm}$ EDTA in $60 \%$ glycerol) and loaded on agarose gel $2 \%$ (containing ethidium bromide $20 \mathrm{ng} / \mathrm{ul}$ ).

$5 \mathrm{ul}$ of 50 bp ready to use DNA ladder (MBI Fermentas) was loaded in a separate lane. Products were visualized on a UV Transilluminator.

C) Restriction digestion:

Restriction digestion was performed at codons 16 and 27 of $\beta 2 \mathrm{AR}$ using Ncol, BsrDI, Bbvl and ItaI restriction enzymes. (24-26) Digestion was performed under the following conditions:

Restriction enzyme: 1 unit

PCR water: 6 ul

Buffer: 3 ul

PCR product: $10 \mathrm{ul}$

Overnight incubation at $37^{\circ} \mathrm{C}$ was followed by gel electrophoresis on 3\% agarose gel. Figure 1-3.

\section{STATISTICAL ANALYSIS}

The distributions of quantitative variables were tested for normality using Kolmogorov-Smirnov test and revealed 
that most of the data were normally distributed, so parametric statistics were applied. Quantitative data were described using mean and standard deviation. Qualitative data as genotypes were described using number and percent.

Association between 2 qualitative variables (2x2) done using Chi square test. This was used for genotypes.

Comparing quantitative variables in 2 groups were conducted using independent sample t test .This was used for pulmonary function parameters. Kruskal Wallis test was used to study the distribution of pulmonary function parameters among the genotype groups.

Correlation between 2 quantitative variables done using pearson correlation test.

In all statistical tests, level of significance of .05 was used, below which the results considered to be statistically significant

Data were analyzed using the Statistical Package for Social Sciences (SPSS ver.20 Chicago, IL, USA)

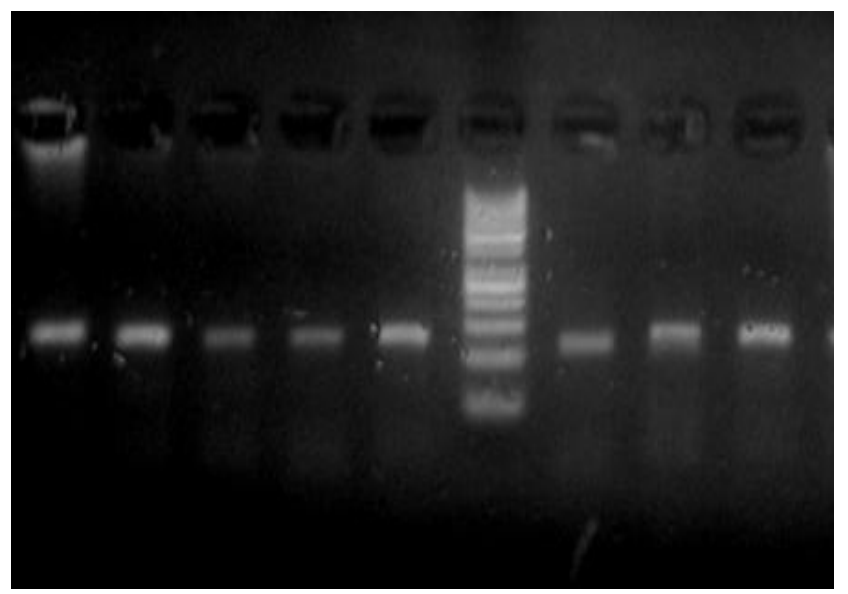

Fig. (1): Lanes 1, 2, 3, 4 and 5: Arg / Arg genotype, Lane 6 : 50 bp DNA ladder, Lane 7 : Gly /Gly genotype, Lanes 8 and 9: Arg / Arg genotype

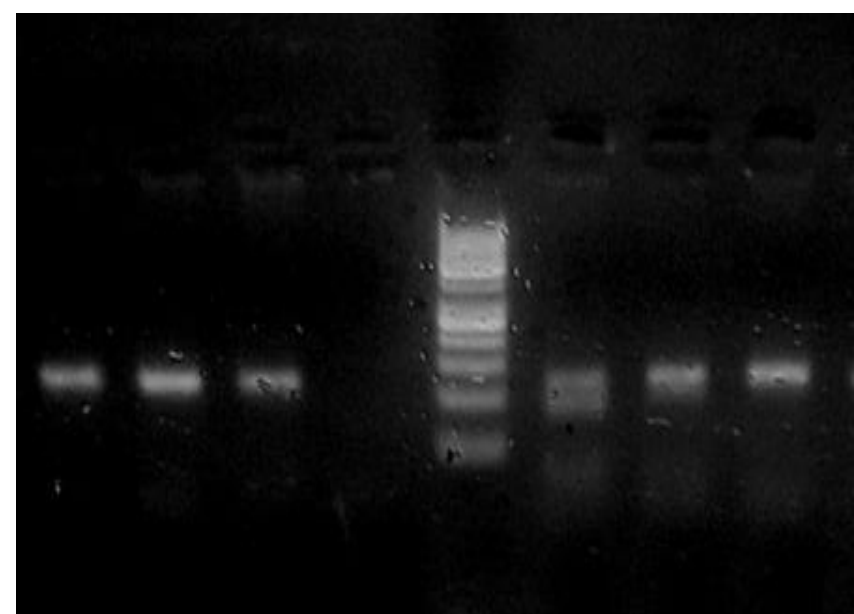

Fig. (2): Lanes 1, 2 and 3: Arg /Arg genotype, Lane 5: $50 \mathrm{bp}$ DNA ladder, Lane 6: Gly /Arg genotype, Lanes 7 and 8: Arg /Arg genotype.

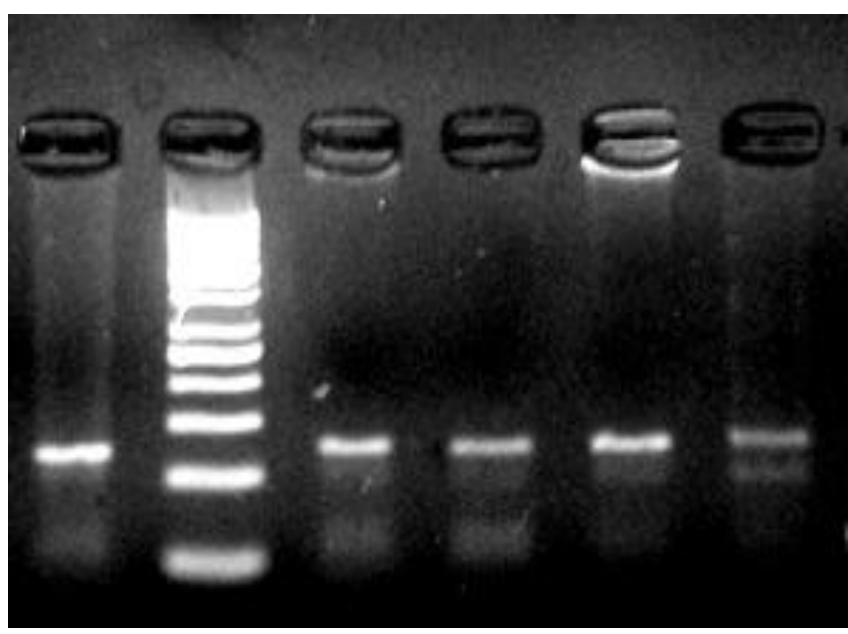

Fig. (3): Lanes 1, 3, 4 and 5: Arg /Arg genotype. Lane 2 : 50 bp DNA ladder, Lane 6: Gly / Arg genotype

\section{RESULTS}

The characteristics of asthmatic patients and controls are presented in table (1). There were no significant difference between patients and control groups as regards the age, weight, and height.Pulmonary function parameters in asthmatic and control subjects are presented in table (1) as actual and percent predicted values. There was a significant difference between the asthmatic and the control subjects in all pulmonary function parameters measured.

The asthmatics were classified according to severity into mild, moderate and severe asthmatics: 24 (40.0\%), 30 $(50.0 \%), 6(10.0 \%)$ respectively.

There was no significant correlation between asthma severity or airway hyper-responsiveness $\left(\mathrm{PD}_{20-\mathrm{FEV} 1}\right)$ and parameters of obesity in the asthmatics Table (2). The Comparison between obese $(\mathrm{n}=30)$ and non-obese asthmatics $(n=30)$ according to AHR $\left(\mathrm{PD}_{20-\mathrm{FEV} 1}\right)$ showed no significant difference between the two groups.

Molecular studies: The Distribution of genotypes frequencies of $\beta_{2} \mathrm{AR}$ gene at codon 16 , codon 27 and codon 16 - codon 27 among obese and non-obese asthmatics (3) and obese and non-obese subjects is illustrated in table (4) which showed a significant association only between the group categories and combined genotype Gly16Arg -Glu27Glu [p $=0.044$, $95 \%$ C.I. $=0.040-0.048]$

The distribution of genotypes frequencies of $\beta_{2} \mathrm{AR}$ gene at codon 16, codon 27 and codon 16 /codon 27 among mild, moderate and severe asthmatics are illustrated in table (5)which revealed a significantly increased frequency of Gly16Gly among severe asthmatics $(66.7 \%)$ [p $=0.021]$ in comparison with the mild $(20.8 \%)$ and the moderate $(13.3 \%)$ asthmatics and a significantly higher frequency of Gly16Gly-Gln27Gln in severe asthmatics (33.3\%) [p = 0.012 ] in comparison to mild $(4.2 \%)$ and moderate $(0 \%)$ asthmatics. 
The pulmonary function parameters in the four studied groups distributed according to genotypes of $\beta_{2} \mathrm{AR}$ gene at codon 16 in table (6) revealed a significant difference in FEV1\%pred $(\mathrm{p}=0.017)$ and in $\mathrm{FEF}_{25-75 \%}$ percent predicted $(\mathrm{p}=0.034)$ in non- obese asthmatics when distributed according to genotypes at codon 16.

The pulmonary function parameters in the four studied groups distributed according to genotypes of $\beta_{2} \mathrm{AR}$ gene at codon 27 showed no significant difference. The pulmonary function parameters in the four studied groups distributed according to combined genotypes of $\beta_{2} \mathrm{AR}$ gene at codon 16-codon 27 showed no significant difference. There was no significant difference in the distribution of the asthmatics according to the value of $\mathrm{PD}_{20-\mathrm{FEV} 1}$ (provocational dose that resulted in $20 \%$ drop in $\mathrm{FEV}_{1}$ ) among genotypes of $\beta_{2} \mathrm{AR}$ gene at codon 16 , codon 27 , and codon 16 - codon 27 .

Table (1): The characteristics and the Pulmonary function parameters for asthmatic and control subjects

\begin{tabular}{|c|c|c|c|}
\hline & $\begin{array}{c}\text { Asthmatics } \\
(n=60)\end{array}$ & $\begin{array}{c}\text { Controls } \\
(n=60)\end{array}$ & $\mathbf{p}$ \\
\hline Male Female & $\begin{array}{l}10(16.7 \%) \\
50(83.3 \%)\end{array}$ & $\begin{array}{l}14(23.3 \%) \\
46(76.7 \%)\end{array}$ & $\mathrm{x} 2 \mathrm{p}=0.361$ \\
\hline $\begin{array}{l}\text { Male : female ratio } \\
\text { Age Mean } \pm \text { SD } \\
\text { Weight }(\mathrm{kg}) \text { Mean } \pm \text { SD } \\
\text { Height }(\mathrm{cm}) \text { Mean } \pm \text { SD } \\
\text { B.M.I. }(\mathrm{kg} / \mathrm{m} 2) \text { Mean } \pm \text { SD }\end{array}$ & $\begin{array}{c}1: 5 \\
33.0 \pm 8.0 \\
80.0 \pm 21.0 \\
164.0-7.0 \\
30.0 \pm 8.0 \\
\end{array}$ & $\begin{array}{c}1: 3.3 \\
34.0 \pm 10.0 \\
82.0 \pm 20.0 \\
165.0 \pm 9.0 \\
30.04 \pm 6.65 \\
\end{array}$ & $\begin{array}{l}0.819 \\
0.554 \\
0.606 \\
0.774 \\
\end{array}$ \\
\hline $\begin{array}{l}\text { Waist circumference }(\mathrm{cm}) \text { Femal } \\
\text { Mean } \pm \text { SD }\end{array}$ & $\mathrm{N}=50,90.72 \pm 15.65$ & $\mathrm{~N}=46,90.24 \pm 13.45$ & 0.873 \\
\hline $\begin{array}{l}\text { Waist circumference }(\mathrm{cm}) \text { Male } \\
\text { Mean } \pm \text { SD }\end{array}$ & $\mathrm{N}=10.92 .50 \pm 13.04$ & $\mathrm{~N}=14,99.86 \pm 18.18$ & 0.287 \\
\hline $\begin{array}{l}\text { Waist / Hip ratio Female } \\
\text { Mean } \pm \text { SD }\end{array}$ & $\mathrm{N}=50,0.83 \pm 0.08$ & $\mathrm{~N}=46,0.84 \pm 0.06$ & 0.302 \\
\hline $\begin{array}{l}\text { Waist / Hip ratio Male } \\
\text { Mean } \pm \text { SD }\end{array}$ & $\mathrm{N}=10,0.89 \pm 0.12$ & $\mathrm{~N}=14,0.90 \pm 0.10$ & 0.858 \\
\hline $\begin{array}{l}\text { FVC }(\text { L) Mean } \pm \text { SD } \\
\text { FVC \% pred. Mean } \pm \text { SD } \\
\text { FEV } 1 \text { (L) Mean } \pm \text { SD } \\
\text { FEV }_{1} \% \text { pred Mean } \pm \text { SD } \\
\text { FEV }_{1} / \text { FVC } \% \text { Mean } \pm \text { SD } \\
\text { FEF }_{25-75 \%}(\text { L/Sec }) \text { Mean } \pm \text { SD } \\
\text { FEF }_{25-75} \% \text { pred. Mean } \pm \text { SD }\end{array}$ & $\begin{array}{c}3.19 \pm 0.64 \\
89.0 \pm 11 \\
2.75 \pm 0.54 \\
89.0 \pm 12.0 \\
87.0 \pm 9.0 \\
2.97 \pm 0.94 \\
76.0 \pm 22.0 \\
\end{array}$ & $\begin{array}{c}3.52^{*} \pm 0.81 \\
96.0^{* *} \pm 12.0 \\
3.24^{* *} \pm 0.81 \\
102.0^{* *} \pm 12.0 \\
92.0^{* *} \pm 6.0 \\
4.17^{* *} \pm 1.38 \\
105.0^{* *} \pm 25.0\end{array}$ & $\begin{array}{c}0.016 \\
0.001 \\
<0.001 \\
<0.001 \\
<0.001 \\
<0.001 \\
<0.001 \\
\end{array}$ \\
\hline
\end{tabular}

FEV1: forced expiratory volume in one second

$\mathrm{FEF}_{25-75 \%}$ : forced expiratory flow between 25 and $75 \%$

FVC: forced vital capacity

$\%$ pred.: percent predicted

$*$ : Statistically significant at $\mathrm{p} \leq 0.05$

$* *$ : Statistically significant at $\mathrm{p} \leq 0.01$

Table (2): The Correlation between Parameters of asthma severity (clinical severity score, airway reactivity score and $\mathrm{FEV}_{1} \%$ pred) and airway hyper-responsiveness $\left(\mathrm{PD}_{20-\mathrm{FEV} 1}\right)$ and the studied parameters of obesity (BMI, waist circumference and waist/hip ratio) in the asthmatics.

\begin{tabular}{|c|c|c|c|c|c|c|c|c|}
\hline & \multicolumn{2}{|c|}{ PD $_{20-\mathrm{FEV} 1}$} & \multicolumn{2}{|c|}{ Clinical severity score } & \multicolumn{2}{|c|}{$\begin{array}{c}\text { Airway reactivity } \\
\text { score }\end{array}$} & \multicolumn{2}{|c|}{ FEV $_{1} \%$ pred. } \\
\hline & $\mathbf{r}_{\mathrm{s}}$ & $\mathbf{p}$ & $\mathbf{r}$ & $\mathbf{p}$ & $\mathbf{r}$ & $\mathbf{p}$ & $\mathbf{r}$ & p \\
\hline BMI $\left(\mathrm{kg} / \mathrm{m}^{2}\right)$ & 0.038 & 0.771 & 0.124 & 0.346 & 0.189 & 0.148 & -0.011 & 0.933 \\
\hline Waist circumference (cm) (male) & -0.320 & 0.367 & 0.217 & 0.546 & 0.321 & 0.366 & -0.574 & 0.083 \\
\hline Waist circumference (cm) (female) & 0.162 & 0.261 & 0.045 & 0.754 & 0.172 & 0.232 & -0.029 & 0.844 \\
\hline Waist /hip ratio (male) & -0.185 & 0.609 & 0.125 & 0.731 & 0.323 & 0.363 & -0.522 & 0.122 \\
\hline Waist /hip ratio (female) & 0.229 & 0.109 & -0.072 & 0.619 & 0.113 & 0.435 & 0.085 & 0.555 \\
\hline
\end{tabular}

r: Pearson coefficient

BMI: Body mass index
$\mathrm{PD}_{20 \mathrm{FEV} 1}$ : Provocational dose of methacholine that resulted in $20 \%$ drop in $\mathrm{FEV}_{1}$ 
Table (3): Distribution of genotypes frequencies of $\beta_{2} \mathrm{AR}$ gene at codon 16, codon 27and codon 16 - codon 27 among obese and non-obese asthmatics.

\begin{tabular}{|c|c|c|c|c|c|c|}
\hline \multirow[b]{2}{*}{ Genotype } & \multicolumn{2}{|c|}{ Asthmatics } & \multirow[b]{2}{*}{ Test of sig. } & \multirow[b]{2}{*}{$\mathbf{p}$} & \multirow[b]{2}{*}{ O.R. } & \multirow[b]{2}{*}{ 95\% C.I. } \\
\hline & $\begin{array}{l}\text { Obese } \\
n=30\end{array}$ & $\begin{array}{c}\text { Non-Obese } \\
\mathbf{n}=\mathbf{3 0}\end{array}$ & & & & \\
\hline \multicolumn{7}{|l|}{ Codon 16} \\
\hline Gly / Gly & $8(26.7 \%)$ & $5(16.7 \%)$ & $x^{2}=0.884$ & 0.347 & 0.550 & $0.157-1.931$ \\
\hline Gly / Arg & $19(63.3 \%)$ & $21(70.0 \%)$ & $x^{2}=0.300$ & 0.584 & 1.351 & $0.460-3.968$ \\
\hline Arg / Arg & $3(10.0 \%)$ & $4(13.3 \%)$ & $\mathrm{FE}$ & 1.000 & 1.385 & $0.282-6.796$ \\
\hline \multicolumn{7}{|l|}{ Codon 27} \\
\hline Gln / Gln & $4(13.3 \%)$ & $8(26.7 \%)$ & $x^{2}=1.667$ & 0.197 & 2.364 & $0.627-8.917$ \\
\hline Gln / Glu & $18(60.0 \%)$ & $19(63.3 \%)$ & $x^{2}=0.071$ & 0.791 & 1.152 & $0.406-3.263$ \\
\hline Glu / Glu & $8(26.7 \%)$ & $3(10.0 \%)$ & $x^{2}=2.783$ & 0.095 & 0.306 & $0.072-1.291$ \\
\hline \multicolumn{7}{|l|}{ Codon 16 - Codon 27} \\
\hline Gly /Gly- Gln / Gln & $2(6.7 \%)$ & $1(3.3 \%)$ & $\mathrm{FE}$ & 1.000 & 0.483 & $0.041-5.628$ \\
\hline Gly / Gly- Gln / Glu & $4(13.3 \%)$ & $3(10.0 \%)$ & $\mathrm{FE}$ & 1.000 & 0.722 & $0.147-3.545$ \\
\hline Gly / Gly- Glu / Glu & $2(6.7 \%)$ & $1(3.3 \%)$ & FE & 1.000 & 0.483 & $0.041-5.628$ \\
\hline Gly / Arg- Gln / Gln & $2(6.7 \%)$ & $6(20.0 \%)$ & FE & 0.254 & 3.500 & $0.645-18.980$ \\
\hline Gly / Arg- Gln / Glu & $13(43.3 \%)$ & $14(46.7 \%)$ & $\chi^{2}=0.067$ & 0.795 & 1.144 & $0.414-3.166$ \\
\hline Gly /Arg- Glu / Glu & $4(13.3 \%)$ & $1(3.3 \%)$ & FE & 0.353 & 0.224 & $0.024-2.136$ \\
\hline Arg / Arg- Gln / Gln & $0(0.0 \%)$ & $1(3.3 \%)$ & FE & 1.000 & 1.034 & $0.968-1.106$ \\
\hline Arg / Arg- Gln / Glu & $1(3.3 \%)$ & $2(6.7 \%)$ & $\mathrm{FE}$ & 1.000 & 2.071 & $0.178-24.148$ \\
\hline Arg / Arg-Glu / Glu & $2(6.7 \%)$ & $1(3.3 \%)$ & FE & 1.000 & 0.483 & $0.041-5.628$ \\
\hline
\end{tabular}

$\mathrm{p}: \mathrm{p}$ value for comparing between the two studied groups

$\chi^{2}$ : Chi square test

FE: Fisher Exact test

Gly : Glycine

Arg: Argnine
Gln: Glutamine

Glu: Glutamic acid

$\%=$ percentage

O.R: Odds ratio

95\% C.I: $95 \%$ confidence interval

Table (4):Distribution of genotypes frequencies of $\beta_{2} A R$ gene at codon 16, codon 27 and codon 16 -codon 27 among obese and non-obese subjects (asthmatics and Non-asthmatics)

\begin{tabular}{lcccccc}
\hline Genotype & $\begin{array}{c}\text { Obese } \\
\mathbf{n = 6 6}\end{array}$ & $\begin{array}{c}\text { Non-obese } \\
\mathbf{n = 5 4}\end{array}$ & Test of sig. & p & O.R. & $\mathbf{9 5 \%}$ C.I. \\
\hline Codon 16 & & & & & & \\
Gly / Gly & $15(22.7 \%)$ & $9(16.7 \%)$ & $\chi^{2}=0.682$ & 0.409 & 0.680 & $0.271-1.704$ \\
Gly / Arg & $45(68.2 \%)$ & $37(68.5 \%)$ & $\chi^{2}=0.002$ & 0.969 & 1.016 & $0.469-2.201$ \\
Arg / Arg & $6(9.1 \%)$ & $8(14.8 \%)$ & $\chi^{2}=0.944$ & 0.331 & 1.739 & $0.564-5.362$ \\
\hline Codon 27 & & & & & \\
Gln / Gln & & & & & \\
Gln / Glu & $9(13.6 \%)$ & $11(20.4 \%)$ & $\chi^{2}=0.970$ & 0.325 & 1.620 & $0.617-4.256$ \\
Glu / Glu & $40(60.6 \%)$ & $37(68.5 \%)$ & $\chi^{2}=0.809$ & 0.369 & 1.415 & $0.663-3.017$ \\
\hline Codon 16 - Codon 27 & $17(25.8 * \%)$ & $6(11.1 \%)$ & $\chi^{2}=4.112$ & 0.043 & 0.360 & $0.131-0.991$ \\
Gly /Gly- Gln / Gln & & & & & & \\
Gly / Gly- Gln / Glu & $3(4.5 \%)$ & $1(1.9 \%)$ & FE & 0.626 & 0.396 & $0.040-3.922$ \\
Gly / Gly- Glu / Glu & $8(12.1 \%)$ & $5(9.3 \%)$ & $\chi^{2}=0.252$ & 0.616 & 0.740 & $0.227-2.408$ \\
Gly / Arg- Gln / Gln & $4(6.1 \%)$ & $3(5.6 \%)$ & FE & 1.000 & 0.912 & $0.195-4.262$ \\
Gly / Arg- Gln / Glu & $5(7.6 \%)$ & $9(16.7 \%)$ & $\chi^{2}=2.382$ & 0.123 & 2.440 & $0.766-7.776$ \\
Gly /Arg- Glu / Glu & $29(43.9 \%)$ & $27(50.0 \%)$ & $\chi^{2}=0.438$ & 0.508 & 1.276 & $0.620-2.626$ \\
Arg / Arg- Gln / Gln & $11(16.7 * \%)$ & $1(1.9 \%)$ & $\chi^{2}=7.243$ & 0.007 & 0.094 & $0.012-0.756$ \\
Arg / Arg- Gln / Glu & $1(1.5 \%)$ & $1(1.9 \%)$ & FE & 1.000 & 1.226 & $0.075-20.076$ \\
Arg / Arg-Glu / Glu & $3(4.5 \%)$ & $5(9.3 \%)$ & FE & 0.465 & 2.143 & $0.488-9.406$ \\
\hline
\end{tabular}

$\chi^{2}$ : Chi square test

FE: Fisher Exact test

$\mathrm{p}$ : $\mathrm{p}$ value for comparing between the two studied groups

*: Statistically significant at $\mathrm{p} \leq 0.05$

Statistically significant at $\mathrm{p} \leq 0.01$
Gly : Glycine

Gln: Glutamine

$\%=$ percentage
Arg: Argnine

Glu: Glutamic acid

O.R: Odds ratio

THE ROLE OF BETA-2 ADRENERGIC RECEPTOR GENE POLYMORPHISM IN THE PATHOPHYSIOLOGY OF ASTHMA ASSOCIATED WITH OBESITY 
Table (5): Distribution of genotypes frequencies of $\beta_{2} A R$ gene at codon 16, codon 27 and codon 16 - codon 27 among asthmatics classified into mild, moderate and severe according to clinical severity score

\begin{tabular}{|c|c|c|c|c|c|}
\hline \multirow[b]{2}{*}{ Genotype } & \multicolumn{3}{|c|}{ Asthmatics } & \multirow[b]{2}{*}{ MCp } & \multirow[b]{2}{*}{ 95\% C.I. } \\
\hline & $\begin{array}{c}\text { Mild } \\
n=24\end{array}$ & $\begin{array}{c}\text { Moderate } \\
\mathbf{n}=\mathbf{3 0}\end{array}$ & $\begin{array}{c}\text { Severe } \\
\mathrm{n}=6\end{array}$ & & \\
\hline \multicolumn{6}{|l|}{ Codon 16} \\
\hline Gly / Gly & $5(20.8 \%)$ & $4(13.3 \%)$ & $4(66.7 \%)^{*}$ & 0.021 & $0.018-0.024$ \\
\hline Gly / Arg & $15(62.5 \%)$ & $23(76.7 \%)$ & $2(33.3 \%)$ & 0.127 & $0.120-0.134$ \\
\hline Arg / Arg & $4(16.7 \%)$ & $3(10.0 \%)$ & $0(0.0 \%)$ & 0.615 & $0.605-0.625$ \\
\hline \multicolumn{6}{|l|}{ Codon 27} \\
\hline Gln / Gln & $6(25.0 \%)$ & $4(13.3 \%)$ & $2(33.3 \%)$ & 0.429 & $0.420-0.439$ \\
\hline Gln / Glu & $15(62.5 \%)$ & $19(63.3 \%)$ & $3(50.0 \%)$ & 0.862 & $0.855-0.868$ \\
\hline Glu / Glu & $3(12.5 \%)$ & $7(23.3 \%)$ & $1(16.7 \%)$ & 0.714 & $0.705-0.723$ \\
\hline \multicolumn{6}{|l|}{ Codon 16 - Codon 27} \\
\hline Gly /Gly- Gln / Gln & $1(4.2 \%)$ & $0(0.0 \%)$ & $2(33.3 \%)^{*}$ & 0.012 & $0.010-0.014$ \\
\hline Gly / Gly- Gln / Glu & $4(16.7 \%)$ & $2(6.7 \%)$ & $1(16.7 \%)$ & 0.615 & $0.605-0.625$ \\
\hline Gly / Gly- Glu / Glu & $0(0.0 \%)$ & $2(6.7 \%)$ & $1(16.7 \%)$ & 0.333 & $0.323-0.342$ \\
\hline Gly / Arg- Gln / Gln & $4(16.7 \%)$ & $4(13.3 \%)$ & $0(0.0 \%)$ & 0.679 & $0.670-0.688$ \\
\hline Gly / Arg- Gln / Glu & $9(37.5 \%)$ & $16(53.3 \%)$ & $2(33.3 \%)$ & 0.493 & $0.483-0.502$ \\
\hline Gly /Arg- Glu / Glu & $2(8.3 \%)$ & $3(10.0 \%)$ & $0(0.0 \%)$ & 1.000 & $1.000-1.000$ \\
\hline Arg / Arg- Gln / Gln & $1(4.2 \%)$ & $0(0.0 \%)$ & $0(0.0 \%)$ & 0.503 & $0.493-0.512$ \\
\hline Arg / Arg- Gln / Glu & $2(8.3 \%)$ & $1(3.3 \%)$ & $0(0.0 \%)$ & 0.698 & $0.689-0.707$ \\
\hline Arg / Arg-Glu / Glu & $1(4.2 \%)$ & $2(6.7 \%)$ & $0(0.0 \%)$ & 1.000 & $1.000-1.000$ \\
\hline
\end{tabular}

MC: Monte Carlo test

*: Statistically significant at $\mathrm{p} \leq 0.05$ by Monte Carlo test Gly : Glycine

Arg: Argnine
Gln: Glutamine

Glu: Glutamic acid

$\%=$ percentage

95\% C.I: $95 \%$ confidence interval

Table (6): The pulmonary function parameters in the four studied groups distributed according to genotypes of $\beta_{2} A R$ gene at codon 16

\begin{tabular}{|c|c|c|c|c|c|c|}
\hline & & & \multicolumn{3}{|c|}{ Codon 16} & \multirow{2}{*}{${ }^{\mathrm{KW}} \mathrm{p}$} \\
\hline & & & Gly/Gly & Gly / Arg & Arg/Arg & \\
\hline \multirow{4}{*}{$\begin{array}{l}\mathrm{FVC}_{(\%)} \\
\text { pred. }\end{array}$} & \multirow[b]{2}{*}{ Asthmatics } & Obese & $88.0(66.50-105.40)$ & $91.50(70.40-116.70)$ & $85.20(83.70-95.50)$ & 0.98 \\
\hline & & Non obese & $86.70(82.40-97.80)$ & $88.40(68.60-103.70)$ & $101.25(82.8-102.4)$ & 0.20 \\
\hline & \multirow[b]{2}{*}{ Controls } & Obese & $105.20(82.7-116.6)$ & $96.35(59.1-111.2)$ & $89.90(87.1-103.7)$ & 0.53 \\
\hline & & $\begin{array}{l}\text { Non } \\
\text { Obese }\end{array}$ & $100.25(79.20-123.0)$ & $93.15(77.0-119.90)$ & $91.75(84.50-106.40)$ & 0.78 \\
\hline \multirow{4}{*}{$\mathrm{FEV}_{1(\%) \text { pred. }}$} & \multirow[b]{2}{*}{ Asthmatics } & Obese & $88.90(72.70-113.80)$ & $84.80(68.10-105.0)$ & $79.90(69.10-104.60)$ & 0.61 \\
\hline & & Non obese & $81.20(70.90-96.10)$ & $87.40(69.10-101.50)$ & $108.85^{*}(88.8-111.8)$ & 0.017 \\
\hline & \multirow[b]{2}{*}{ Controls } & Obese & $109.80(75.10-128.8)$ & $99.75(70.9-122.3)$ & $94.10(85.2-119.1)$ & 0.59 \\
\hline & & Non obese & $\begin{array}{c}104.75(90.40- \\
123.30)\end{array}$ & $103.75(88.40-123.50)$ & $100.15(88.2-112.6)$ & 0.88 \\
\hline \multirow{4}{*}{$\begin{array}{l}\mathrm{FEF}_{25-75 \%} \\
\text { (\%)pred. }\end{array}$} & \multirow[b]{2}{*}{ Asthmatics } & Obese & $79.90(62.20-104.30)$ & $80.30(41.30-105.50)$ & $60.70(27.70-92.10)$ & 0.57 \\
\hline & & Non obese & $56.40(44.20-86.90)$ & $77.50(39.40-115.0)$ & $102.0^{*}(73.80-111.30)$ & 0.03 \\
\hline & \multirow[b]{2}{*}{ Controls } & Obese & $93.30(56.90-145.50)$ & $104.1(63.6-152.3)$ & $94.0(69.30-135.8)$ & 0.96 \\
\hline & & Non obese & $96.15(91.0-102.8)$ & $119.30(72.39-155.5)$ & $94.05(70.0-142.7)$ & 0.30 \\
\hline
\end{tabular}

Values are expressed as median (Min. - Max.)

*: Statistically significant at $\mathrm{p} \leq 0.05$
${ }^{\mathrm{KW}} \mathrm{p}$ : $\mathrm{p}$ value for Kruskal Wallis test for comparing between the studied groups $\mathrm{n}=30$ in all groups 


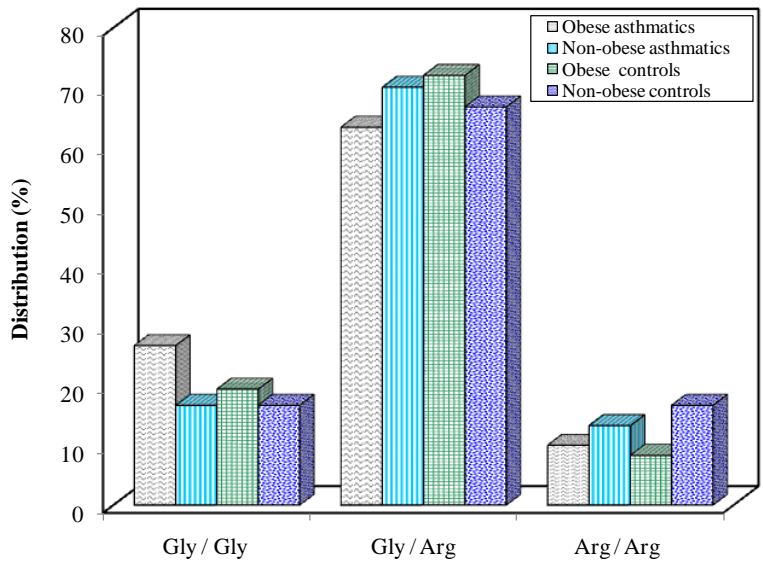

Codon 16

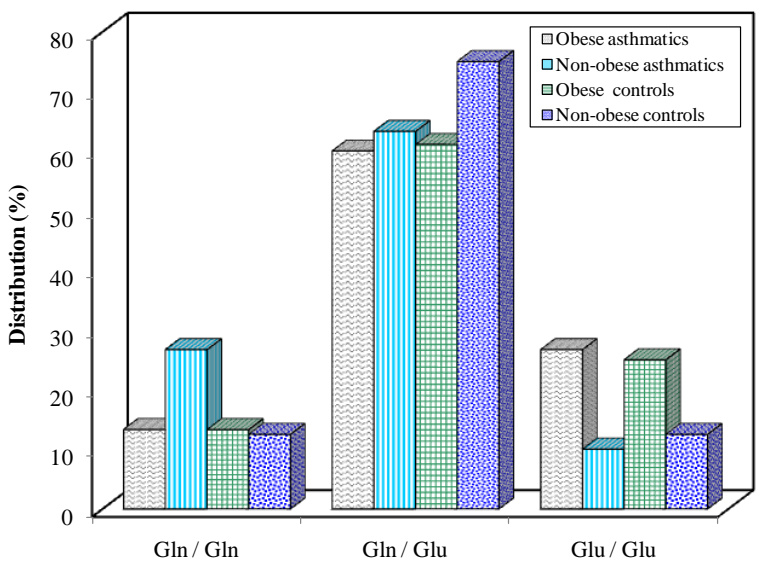

Codon 27

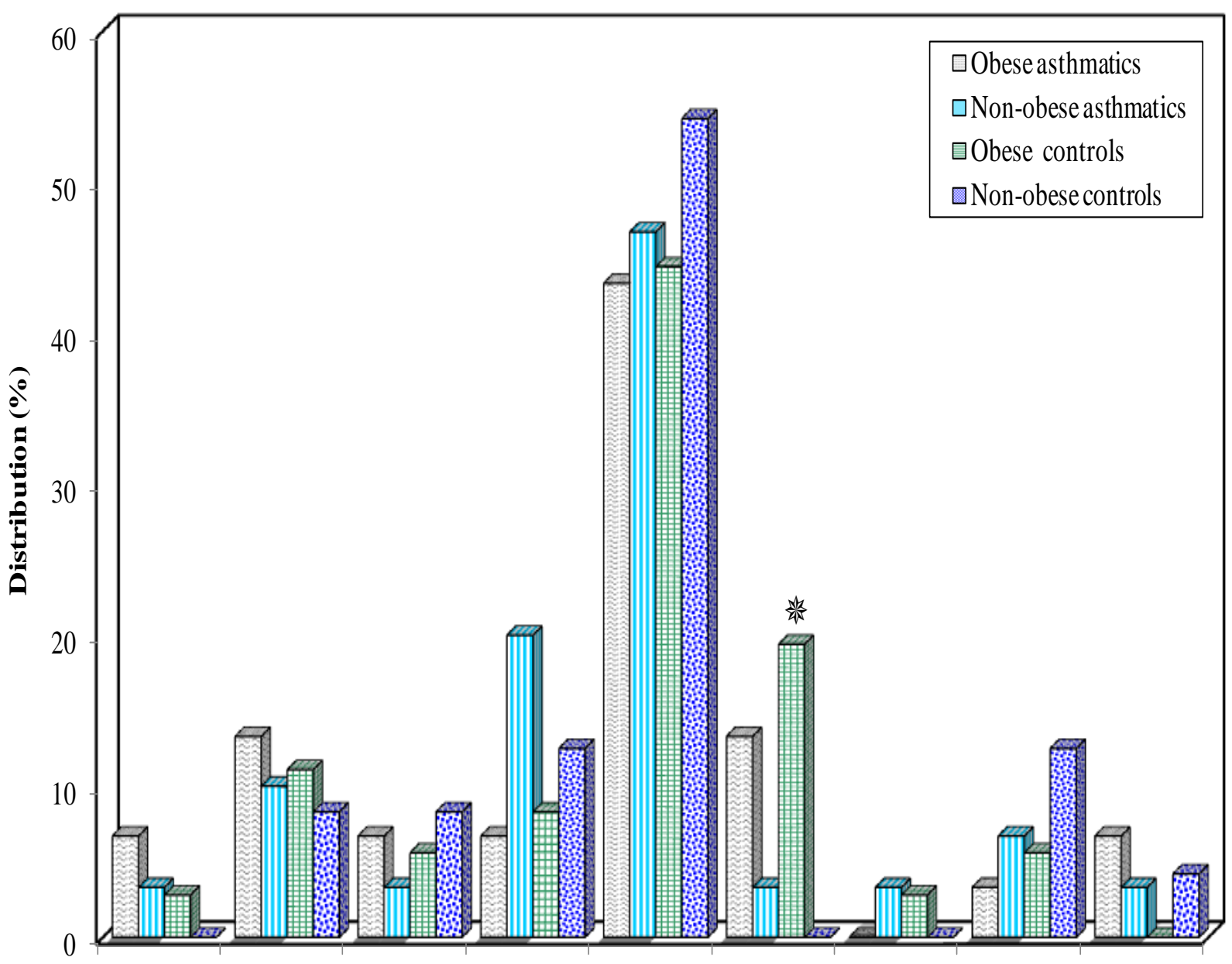

Gly/Gly- Gly/Gly- Gly/Gly- Gly/Arg- Gly/Arg- Gly/Arg- Arg/Arg- Arg/Arg- Arg/ArgGln/Gln Gln/Glu Glu/Glu Gln/Gln Gln/Glu Glu/Glu Gln/Gln Gln/Glu Glu/Glu

\section{Codon 16 - Codon 27}

Figure (4): Distribution of genotypes frequencies of $\beta_{2} \mathrm{AR}$ gene at codon 16, codon 27 and codon 16-codon 27 among obese and non-obese asthmatics and obese and non-obese control subjects 


\section{DISCUSSION}

In the current study no significant difference could be detected among the asthmatic and control subjects in the distribution of allelic or genotypes frequencies of $\beta 2 \mathrm{AR}$ gene at codon 16 and codon 27. Also, there was no significant difference could be detected in the distribution of the asthmatics (according to the value of $\mathrm{PD}_{20-\mathrm{FEV} 1}$ ) among genotypes of $\beta 2 \mathrm{AR}$ gene at codon 16 and codon 27. However, a significantly increased frequency of genotype Gly16Gly was observed among severe asthmatics in comparison with mild and moderate asthmatics. Yet, there was no significant genotype that associated asthma and obesity.

In the present study, there was no significant difference in the distribution of allelic or genotype frequencies of $\beta 2 \mathrm{AR}$ gene at codon 16 and codon 27 in asthmatics when compared to control subjects. This finding is in agreement with the findings of other investigators. ${ }^{.27-30)}$

However this is contradicted in a study by Salah $\mathrm{K}$ et al,2012 ${ }^{(31)}$ which found higher frequencies of Arg16Gly and Gly16Gly genotypes in asthmatic children when compared with controls, and a study ${ }^{(32)}$ that reported an association between $\beta 2 \mathrm{AR}-16$ and $\beta 2 \mathrm{AR}-27$ gene polymorphisms with asthma diagnosis and nocturnal symptoms among Mexican Mestizos, These results suggested that variation in the $\beta 2 \mathrm{AR}$ gene is associated with asthma pathogenesis and may act as a disease modifier in nocturnal asthma, and found an association among men with the "Gly16 allele without Glu27" and asthma diagnosis. They emphasize to the necessity of accounting for ethnic background in performing $\beta 2 \mathrm{AR}$ genotyping. Interethnic differences in the $\beta 2 \mathrm{AR}$ structure could explain differences in the incidence and prevalence of asthma, disease susceptibility, asthma severity, and treatment response. Assessment of the sex difference which could be of major importance in $\beta 2 \mathrm{AR}$ function and its role in the pathogenesis of asthma should be considered. The difference could also be explained by gene-environment interactions with $\beta 2 \mathrm{AR}$ gene polymorphisms. ${ }^{(32)}$

The present study showed that GLy16Gly genotype and the combined genotype Gly16Gly-Gln27Gln were significantly elevated in severe asthmatics in comparison with mild and moderate asthmatics. No significant association was found between polymorphism at codon 27 and asthma severity. This was in consistence with several studies ${ }^{(31,33-35)}$ However, Palmer et al., $\left({ }^{36)}\right.$ revealed that the arginine-16 genotype of ADRB2 predisposes to exacerbations in asthmatic children and young adults. In addition Reihsaus et al., ${ }^{(17)}$ found no correlation between Gly16 homozygosity and hospital admissions, and Wier et al., ${ }^{(37)}$ found no increase in frequency of Gly16 in fatal or no fatal asthma. So it seems that various host and environmental factors other than polymorphism can affect the severity of asthma; factors such as poor economic condition, lower level of education and concomitant diseases. ${ }^{(38)}$ Allergy was found as another significant predictor, which proves that the positive history of allergy is a risk factor of asthma severity. ${ }^{(39)}$

Several studies (40-42) supported the current study whichdetected significant difference among non-obese asthmatic patients in FEV1 \%predicted when distributed according to genotypes at codon 16 of the ADRB2; there was a significant increase in FEV1\%predicted in Arg16 homozygotes. This is supported by In the present study there was a significant difference in proportion of combined genotype Gly16Arg-Glu27Glu among the four studied groups, however this was mainly due to theeffect of obesity rather than asthma.

\section{Limitations}

Our findings have led us to expect that studies of genegene and gene-environment interactions and epigenetic mechanisms can help us understand the link between asthma and obesity. Because the $\beta$-receptor is only one element affecting $\beta$-agonist response, gene-gene interactions must be addressed to assess biological pathways that affect $\beta$ - agonist response. The complex interrelationship between obesity and asthma is an example of how genes and environment interact. Through inflammatory mechanisms or lifestyle changes, obesity may give rise to asthmatic symptoms in susceptible individuals. The interaction between diet and genes may cause abnormalities in body growth patterns (leading to obesity) and/or alter airway tone (triggering asthma), and multiple interrelations between obesity and asthma may then take place.

\section{Conclusion:}

In conclusion Gly16Gly genotype was associated with increased asthma severity. Arg16Arg genotype was found to be protective from development of severe asthma. However, there was no detectable specific genotype for the association of asthma and obesity.

\section{REFERENCES}

1. Beuther D, Weiss S, Sutherland E. Obesity and asthma. Am J Respir Crit Care Med 2006;174:112-9.

2. Shore S. Obesity and asthma: Possible mechanisms. J Allergy Clin Immunol 2008; 121(5):1087-93.

3. Bustos P, Amigo H, Oyarzun M, Rona R. Is there a causal relation between obesity and asthma? Evidence from Chile.Int J ObesRelatMetab Disord 2005; 29 (7): 804-9

4. Ford E. The epidemiology of obesity and asthma. J Allergy ClinImmunol 2005; 115 (5):897-909.

5. Shore S, Johnston R. Obesity and asthma. PharmacolTher 2006; 110 (1):83-102

6. Shore S. Obesity and asthma: implications for treatment. CurrOpinPulm Med 2007;13 (1):56-62.

7. Shore S. Obesity and asthma: lessons from animal models. J ApplPhysiol 2007; 102 (2):516-28.

8. Ober C, Hoffjan S. Asthma genetics: the long and winding road to gene discovery. Genes Immun 2006; 7: 95-100. 
9. Rankinen T, Zuberi A, Chagnon Y, et al. The human obesity gene map: the 2005 update. Obesity (Silver Spring) 2006; 14: 529-644.

10. Castro-Giner F, Kogevinas M, Imboden M, et al. Joint effect of obesity and TNFA variability on asthma: two international cohort studies. Eur Respir J 2009; 33:1003-9.

11. Murphy A, Tantisira K, Soto-Quiros M, et al. PRKCA: a positional candidate gene for body mass index and asthma. Am J Hum Genet 2009; 85:87-96.

12. Szczepankiewicz A, Breborowicz A, Sobkowiak P, Popiel A. Are genes associated with energy metabolism important in asthma and BMI? J Asthma 2009; 46:53-8.

13. Beaudet A. 1998 ASHG presidential address. Making genomic medicine a reality. Am J Hum Genet 1999; 64(1):1-13

14. Litonjua A, Gong L, Duan Q, et al. Very important pharmacogene summary ADRB2. Pharmacogenet Genomics 2010; 20: 64-9.

15. Bachman E, Dhillon $\mathrm{H}$, Zhang $\mathrm{C}$, et al. Beta AR signaling required for diet-induced thermogenesis and obesity resistance. Science 2002; 297:843- 5.

16. Hupfeld C, Dalle S, Olefsky J. Beta-Arrestin 1 downregulation after insulin treatment is associated with supersensitization of beta 2 adrenergic receptor $\mathrm{G}$ alpha s signaling in 3T3-L1 adipocytes. ProcNatlAcadSci U S A $2003 ; 100: 161-6$.

17. Reihsaus E, Innis M, MacIntyre N, Liggett S. Mutations in the gene encoding for the $\beta 2$-adrenergic receptor in normal and asthmatic subjects. Am J Respir Cell MolBiol 1993; 8: 334-9.

18. Jalba M, Rhoads G, Demissie K. Association of Codon 16 and Codon $27 \quad \beta 2$-Adrenergic Receptor Gene Polymorphisms with Obesity: A Metaanalysis. Obesity 2008; 16: 2096-06.

19. American Thoracic Society. Standards for the diagnosis and care of patients with chronic obstructive pulmonary disease (COPD) and asthma. AM Rev Respir Dis1987; 136: 225-44.

20. Brooks SM, Bernstein L, Raghuprasad PK, MacciaC,Mieczkowski L. Assessment of airway hyperresponsivenes in chronic stable asthma. J Allergy Clin Immunol.1990; 85: 17-26.

21. Pellegrino R, Viegi G, Brusasco G, et al. Interpretative strategies for lung function tests series "ATS/ERS task force: standardisation of lung function testing". EurRespir J 2005; 26: 948-68.

22. American Thoracic Society. Guidelines for methacholine and exercise challenge testing. Am J RespirCrit Care Med 2000; 161: 309-29.

23. Miller S, Dykes D, Polesky H. A simple salting out procedure for extracting DNA from human nucleated cells. Nucleic Acids Res 1988; 16: 1215.

24. Martinez F, Graves P, Baldini M, Solomon S, Erickson R. Association between genetic polymorphisms of the $\beta 2$-adrenoceptor and response to albuterol in children with and without a history of wheezing. J Clin Invest 1997; 100: 3184-8

25. Large V, Hellstrom L, Reynisdottir S. Human beta-2 adrenoceptor gene polymorphisms are highly frequent in obesity and associate with altered adipocyte beta- 2 adrenoceptor function. Journal of Clinical Investigation 1997; 100(12): 3005-13.

26. Litonjua A, Silverman E, Tantisira K. $\beta 2$-adrenergic receptor polymorphisms and haplotypes are associated with airways hyperresponsiveness among nonsmoking men. Chest 2004; 126: 66-74.

27. Pagaria M. Beta 2 adrenoreceptor polymorphisms (ADR gene) are not strongly associated with asthma incidence or prevalence. Thorax 2007; 62:28.

28. Isaza C, Sepúlveda-Arias J, Agudelo B, et al. B2Adrenoreceptor Polymorphisms in asthmatic and nonasthmatic schoolchildren from colombia and their relationship to treatment response. Pediatric Pulmonology 2012; 47(9): 848-55.

29. Contopoulos-Ioannidis D, Manoli E, Ioannidis J. Metaanalysis of the association of b2-adrenergic receptor polymorphisms with asthma phenotypes. J Allergy ClinImmunol 2005; 115: 963-72.

30. El- Akkary I, El-Kholy Z, Mokhtar M, Mostafa M, Masoud I, Adam A. Study of the possible role of $\beta 2$ adrenergic receptor gene polymorphism in the pathogenesis of bronchial hyperresponsiveness in asthmatic patients and its relation to disease severity and treatment response. Journal of American science 2012; 8(10):394-408.

31. Salah K, Morsy S, Atta A. Effects of $\beta 2$-adrenergic receptor polymorphisms on asthma severity and response to salbutamol in Egyptian children. Egypt $\mathbf{J}$ Pediatr Allergy Immunol 2012; 10(2):81-6.

32. Santillan A, Carlos A. Camargo J, Rivera A, et al. Association between $\beta 2$ - adrenoceptor polymorphisms and asthma diagnosis among Mexican adults. J Allergy ClinImmunol 2003; 112;1095-100.

33. Yin K, Zhang X, Qiu Y. Association between beta2adrenergic receptor genetic polymorphisms and nocturnal asthmatic patients of Chinese Han nationality. Respiration 2006; 73(4):464-7.

34. Shigemitsu H, Afshar K. Nocturnal asthma. CurrOpinPulm Med 2007; 13(1):49-55.

35. Hizawa N. Pharmacogenetics of $\beta 2$-agonists. AllergolInt 2011; 60(3):239- 46

36. Palmer C, Lipworth B, Lee S, Ismail T, Macgregor D, Mukhopadhyay S. Arginine-16 b2 adrenoceptor genotype predisposes to exacerbations in young asthmatics taking regular salmeterol. Thorax 2006; 61: 940-4

37. Weir T, Mallek N, Sandford A. Beta2-adrenergic receptor haplotypes in mild, moderate and fatal/near fatal asthma. Am J RespirCrit Care Med 1998; 158:791.

38. Bateman E, Hurd S, Barnes P, et al. Global strategy for asthma management and prevention: GINA executive summary. EurRespir J 2008; 31: 78-143.

39. Salamzadeh J, Wong I, Hosker H, Chrystyn H. A logistic regression analysis of predictors for asthma hospital re-admissions. Iranian J Pharm Res 2003; 5-9.

40. Kim S, Ye Y, Hur G, et al. Effect of beta2-adrenergic receptor polymorphism in asthma control of patients receiving combination treatment. Yonsei medical journal. 2009; 50(2):182-8.

41. Salama M, Ashaat N, Hamad A. Genetic association between common beta-2 adrenoreceptor polymorphism and asthma severity in school-age children. Egyptian Journal of Medical Human Genetics 2011; 12(2): 151-6.

42. Basu K, Palmer C, Tavendale R, Lipworth B, Mukhopadhyay S. Adrenergic beta2-receptor genotype predisposes to exacerbations in steroid-treated asthmatic patients taking frequent albuterol or salmeterol. J Allergy Clin Immunol 2009; 124(6):1188-94. 